\title{
Cerebral Palsy In Children: Subtypes, Motor Function And Associated Impairments; Addis Ababa, Ethiopia
}

Selamenesh Tsige ( $\square$ selidoc2@gmail.com )

Addis Ababa University

Ayalew Moges

Addis Ababa University

Amha Mekasha

Addis Ababa University

Workeabeba Abebe

Addis Ababa University

Hans Forssberg

Karolinska Institutet and Astrid Lindgren Children's Hospital

\section{Research Article}

Keywords: Cerebral Palsy, children, subtypes, impairments, motor function, Ethiopia

Posted Date: May 4th, 2021

DOI: https://doi.org/10.21203/rs.3.rs-460406/v1

License: (9) This work is licensed under a Creative Commons Attribution 4.0 International License. Read Full License

Version of Record: A version of this preprint was published at BMC Pediatrics on December 1st, 2021. See the published version at https://doi.org/10.1186/s12887-021-03026-y. 


\section{CEREBRAL PALSY IN CHILDREN: SUBTYPES, MOTOR FUNCTION AND ASSOCIATED IMPAIRMENTS; ADDIS ABABA, ETHIOPIA}

Selamenesh Tsige (1), Ayalew Moges (1), Amha Mekasha (1), Workeabeba Abebe (1), Hans Forssberg (2)

Correspondence to [selidoc2@gmail.com].

1. Department of pediatrics and child health, College of Health Sciences, Addis Ababa University

2. Department of Women's and Children's Health, Karolinska Institutet and Astrid Lindgren Children's Hospital, Stockholm, Sweden

\section{Abstract}

\section{Background:}

Cerebral Palsy is one of the most common developmental disabilities among children seeking health care services in Ethiopia as well as in other low income countries. Yet, there is no national data relating to CP in Ethiopia. The overall aim of this study is to describe the clinical subtype, gross and fine motor function and presence and pattern of associated impairments and possible risk factors in children with Cerebral Palsy aged 2 to 18 years in Tikur Anbessa Specialized Hospital, Addis Ababa, Ethiopia.

Methods: A hospital based descriptive cross-sectional study conducted among 207 children with Cerebral Palsy. Surveillance for Cerebral Palsy in Europe (SCPE) decision tree was used to include 174 illegible children then a pretested and pre-coded questionnaire administered to caregivers assessing socio demographic characteristics, associated impairments and possible risk factors; evaluated with proper neurologic examination to classify them; and severity of gross and fine motor impairment was assessed by evaluating level of function by grading according to GMFCS and MACS. The IBM SPSS V.21 was used for data analysis, and a significance level of 0.05 was chosen.

Result: A total of 174 children who fulfilled the clinical criteria were included sampled consecutively. Mean age 5.6 (SD 3.6) year; Males 55.2\%. Under five children were 50.6\%. Majority of the children had Bilateral spastic CP (60.4\%) followed by unilateral spastic CP $21.8 \%$, Dyskinetic CP 10.4\%, Ataxic CP $3.4 \%$ and $4 \%$ were unclassifiable. Of all the children $95.4 \%$ had speech and language impairments, $87.4 \%$ learning disabilities; $60.9 \%$ epilepsy, $24.7 \%$ Visual impairment and $8.6 \%$ has hearing impairment. On gross motor functions and manual ability assessment $75.3 \%$ of the children had level 4and 5 functional impairment. More than $80 \%$ of the mothers had complications during delivery (fetal distress followed by PROM). Half of the neonates did not cry immediately after birth; 77 neonates were resuscitated with BMV at birth and 111 immediately admitted to NICU. Subsequently during the first month of life; 50\% had infection, $62 \%$ trouble feeding, $49.4 \%$ difficulty breathing, 35\% seizure and $13.8 \%$ had deep jaundice.

Conclusion; the severe forms of $\mathrm{CP}$ prevail and most children are dependent on their parents for routine activities and cannot communicate well. Multidisciplinary care approach and focused functional rehabilitation services need to be instituted. Causal relationship cannot be drawn from this study but makes a strong argument for improving maternal and child health care.

Key Words: Cerebral Palsy; children; subtypes; impairments; motor function; Ethiopia 


\section{Background}

Cerebral Palsy (CP) is one of the most common developmental disabilities among children seeking health care services in Ethiopia as well as in other low and middle income countries (LMIC). Yet, it was not included in a recent report on the Global Burden of Disease of Developmental Disabilities. ${ }^{1}$ One reason for this omission, is probably the lack of robust population based studies in Africa and other LMIC. Until recently there were only studies on clinical samples suggesting a wide range of prevalence estimates from 2-10 cases per 1000 children $^{2-5}$. During recent years, a couple of rigorous population based studies have been published from Uganda ${ }^{6}$ and Bangladesh ${ }^{7}$ revealing large discrepancies compared with prevalence data from $\mathrm{HIC}^{8}$. The Ugandan and Bangladesh studies showed higher prevalence of CP 2.9/3.4 per 1000 children, compared to about 2 per 1000 in HIC. In fact, during the first years of life the prevalence was even higher, declining over time by a high premature mortality after the first years ${ }^{6}$. In addition to having a higher prevalence, the etiological risk factors differed in Uganda, with almost no preterm born children (compared to $<40 \%$ in HIC), and numerous cases due to post neonatal infections (e.g., malaria). These studies clearly show the need of studies on CP from LMIC and that information cannot be extrapolated from studies in HIC.

A population based study in Uganda revealed that Children with CP in Uganda lack access to health care, Assistive devices, and education, which probably contributes to their poor development of mobility and self-care skills ${ }^{9}$. A national survey on children with disabilities in Ethiopia suggests that the vast majority are not in school, and anecdotal evidence suggests many do not have access to community-based rehabilitation $^{10,11}$. However, the prevalence of children and adults with disability, including $\mathrm{CP}$, is not known in Ethiopia, and there is no studies describing the clinical and socio demographic panorama of children with CP. It is not known what subtypes of $\mathrm{CP}$ are present, the level of motor function these children exhibit, or the presence of risk factors causing the disorder. The aim of this study was to describe clinical subtypes, motor impairments and associated impairments and risk factors using comprehensive methodology and terminology of a clinical cohort of children with CP at the University Hospital in Addis Ababa, the capital of Ethiopia. The results will increase our knowledge on the situation of CP in Ethiopia, and serve as a basis for setting up larger epidemiological and community-based intervention studies to improve the quality of life of children with $\mathrm{CP}$ and their caregivers in Ethiopia. 


\section{Methods}

\section{Study Setting}

This was a prospective, hospital based, descriptive cross-sectional study conducted July-September 2018, at the Pediatrics Neurology Outpatient Clinic in Tikur Anbessa Specialized Hospital (TASH), Department of Pediatrics and Child Health, Addis Ababa. TASH is the largest teaching referral hospital in Ethiopia with over 700 beds. About 500-700 children visit the Pediatrics Neurology Outpatient Clinic every month; of these 25-30\% have cerebral palsy; two third of the patients are from Addis Ababa and the rest are referred from countryside with the majority coming from Oromia region.

\section{Participants and procedures}

A total of 174 children in the age span 2 - 18 years with confirmed diagnosis of $\mathrm{CP}$ were included in the study. They were recruited from a total of 207 children from two groups with suspect motor symptoms. In the first group, 31 children were searching the neurological outpatient clinic for the first time, and in the second group, we re-examined 176 children who had earlier visited the clinic and been diagnosed with CP by less stringent diagnostic procedures.

The assessments were conducted in a series of three steps performed by the principal investigator (ST) and two General Practitioners (GP). In the first step, ST screened all children in both groups using targeted history, physical examination and chart review. Children with obstructive hydrocephalus $(\mathrm{n}=2)$, extra cerebral birth defects $(n=2)$, progressive motor disorder $(n=11)$, muscle hypotonia $(n=7)$ or presence of an isolated spinal neural tube defect $(n=3)$ were excluded. In total 25 children, 11 from first group and 14 from the second group, were excluded.

In the second step, 182 children (20 from the first group and 162 from the second group) were examined by the GPs according to the SCPE decision tree ${ }^{8}$. All 20 children from the first group were confirmed to have $\mathrm{CP}$, while 8 children from the second group, did not fulfill the criteria for $\mathrm{CP}$. Among the eight who were excluded, three had only posture or movement abnormalities but no motor function abnormality, four had loss of previously acquired skills, and one had generalized muscle hypotonia without symptoms of ataxia.

In the third step, the 174 children with confirmed CP included in the study population were assessed by structured questionnaire and neurologic examination with the tools described below and classified to subtype $\mathrm{CP}^{8}$. The above stated procedures and steps followed based on the SCPE decision tree to select the study participants is clearly outlined on Figure 1.

1. Socio demographic

The GPs used a pretested and pre-coded questionnaire to interview the caregivers. Questions include the child's age, sex, level of education, medical history from pregnancy to the present, family history, the 
child's developmental milestones, past and present nutritional history, parental educational status, living condition and family history of illness.

\section{General Clinical Examination}

A standard neurological examination including assessment of motor impairment was performed by the GPs advised by pediatric neurologist (AM) and ST.

\section{Functional Assessment}

Gross and fine motor functions were assessed and classified according to the GMFCS and MACS by the GPs. Theses classification systems grade the motor impairments from mild to severe in a 5 level Likert scale, where Level I indicates mild limitations and V severe impairments.

\section{Associated impairments}

Information of associated impairments were collected by structured interviews with the caregiver using a protocol based on the UNICEF/ Washington group Child functioning module. The associate impairments assessed for included Intellectual disability, Behavioral abnormalities, Speech difficulties, Feeding difficulties, Visual impairment and Hearing impairment and seizures (see supplement info for details).

\section{5. $\underline{\text { Risk factors }}$}

Information on prenatal, perinatal and postnatal risk factors were assessed using structured questionnaires

administered to caregivers. The questions addressed any complications during the antenatal period such as maternal infections and drug intake, as well as gestational age, place of delivery, birth weight, and complications during delivery, neonatal resuscitation, neonatal admissions and medical history during the first 2 years of life.

Quality control; The principal investigator daily checked for completeness of data collection, verified physical examination finding and classification of subtype of $\mathrm{CP}$ for each child.

\section{Data analysis}

After data cleaning and entry, analysis was done using the Statistical Package for Social Sciences (SPSS) version 21 by an employed biostatistician. Data cleaning and entry was also done manually by the principal investigator and cross checked with the entered data by the biostatistician. The data was analyzed using descriptive statistics and the statistical significance was tested using chi square. $\mathrm{P}$ value less than 0.05 was considered as statistically significant. Bivariate analysis was done to see crude association followed by multivariate analysis for significantly associated factors. 


\section{Results}

\section{Clinical subtypes and severity of gross and fine motor impairments}

Bilateral spastic CP was the predominant subtype (60.4\%) followed by Unilateral spastic CP (21.8\%), Dyskinetic CP (10.4\%), Ataxic CP (3.4\%) and unclassifiable CP (4\%). Among children with bilateral spastic CP, 88 children (84\%) had quadriplegic. Left sided involvement was predominant among children with unilateral spastic $\mathrm{CP}$.

The distribution among GFMCS and MACS levels severity of the motor function impairments are shown in Table 1. A majority of children presented with severe impairments in both gross motor $(75 \%$ at GMFCS level IV-V) and fine motor functions (75\% at MACS level IV-V). Only 14\% had the milder levels (I-II) of impairments.

\section{Associated Impairments}

The prevalence of the recorded associated impairments is shown in table 2. Each child had at least one associated impairment and 30 children had two and 144 three or more. Speech difficulties (95\%) and intellectual disability (87\%) were most common. Seizure was present in $61 \%$ of the children, and of these $85 \%$ had been medicated with anticonvulsant, and $90 \%$ had been taken to the "holy water" (traditional healing spiritual water) at least one time. Seizure was also the most common cause of admission to hospital. Visual and hearing impairments were less prevalent, yet quite common, $24.7 \%$ and $8.6 \%$, respectively. All children with visual or hearing impairments were sent to specialist, and $20.7 \%$ and $4.6 \%$, respectively were confirmed. The remaining children did not show up at their appointed time.

The statistical analysis revealed association between sub-type, GMFCS and MACS severity levels and some associated impairments. There was a strong correlation between speech/language impairment (Pvalue - 0.007) and the subtype of $\mathrm{CP}(\mathrm{P}$-value -0.001$)$. Multivariate analysis showed that children with language impairment were 11.5 times more likely to be at GMFCS level IV-V compared with those without language impairment. Children with bilateral spastic CP were 3 times more likely to have GMFCS IV-V, compared to other subtypes. Bivariate analysis, showed a correlation between speech and language impairment, visual impairment and GFMCS and MACS levels. However, on multivariate analysis, the only association that remained with statistical significance was the association between GMFCS and MACS. The subtypes of CP were not associated with MACS levels. 


\section{Perinatal risk factors}

Information about the antenatal, perinatal and postnatal period is presented in table 3 . There were little remarkable events during the antenatal care: In more than half of the mothers the duration of labor was less than 24 hours. Notably, less than $10 \%$ of the children in this CP cohort were born preterm and none had a birth weight below 1000g.

However $95 \%$ of the mothers had complications during delivery identified as fetal distress $(42.7 \%)$ followed by prolonged rupture of membrane (PROM) (25.9\%). Almost two thirds of the children had been admitted to the NICU; most common cause of admission was respiratory distress (76.6\%) and infection $(11.7 \%)$. In the postnatal period, $54 \%$ of the neonates had infection (4.2\% meningitis and 3.2\% tetanus), $62 \%$ had trouble feeding, $49.4 \%$ had difficulty breathing, 35\% had seizure and $13.8 \%$ had deep jaundice with acute bilirubin encephalopathy.

The Bivariate analysis of perinatal factors, showed that the presence of fetal distress and PROM were associated with higher frequency of spastic cerebral palsy subtypes, whereas precipitated labor was associated with higher frequency of dyskinetic or ataxic forms of cerebral palsy. The requirement for bag mask ventilation was associated with higher prevalence of spastic cerebral palsy. The presence of seizure in the neonatal age was more common in spastic cerebral palsy, while deep jaundice was associated with higher prevalence of dyskinetic/ataxic subtypes. There was no association identified between the perinatal factors and the motor function impairment severity level. The multivariate analysis is shown on table 4.

\section{Sociodemographic characteristics}

Details of sociodemographic characteristics is presented on Table 5; The mean age of the 174 children in the CP cohort was 5.6 (SD 3.6) years with male predominance. Seventy percent of the children above 5 years did not attend school, and among those who attended one third performed on the last ten ranks in the respective classroom and $20 \%$ had repeated a grade. Mothers alone were the primary care givers in $24.7 \%$ of the children, $56.9 \%$ of children were being cared by both parents (mothers being the primary caregivers supported by fathers). Majority of the mothers (70.4\%) were house wives.

\section{Discussion}

This clinical cohort of children with confirmed $\mathrm{CP}$ diagnosis from the main tertiary hospital in Ethiopia included a large majority of severely motor impaired children with associated impairments and seizures. In most children, one or several perinatal risk factors were identified, unmasking the potential to prevent 
the brain injury if properly treated. A majority of children did not attend school but was cared for by the mothers working as house wives.

\section{Comparison with other clinical and population based cohorts}

The clinical profile of this cohort differs from recent population based cohorts of children with $\mathrm{CP}$ in Uganda $^{6}$ and Bangladesh ${ }^{7}$. We found that $75 \%$ of children had severe motor impairments at GMFCS or MACS level IV-V, while children with such severe impairments ranged between 36-48 \% in the Ugandan and Bangladesh cohorts, and 32-33\% in population cohorts from $\mathrm{HIC}^{8,12}$. In our cohort, we found few children with mild impairments (GMFCS level I-II, which was prevalent particularly among the older children in the Ugandan cohort. The high proportion of children with severe impairments are more in par with other clinical cohorts from Uganda, Egypt ${ }^{4,5,13,14,15}$, North India ${ }^{16}$. Many of the children suffered from seizures (61\%), and had probably been identified when seeking to get anti-convulsing drugs.

The rate of speech and language impairment in our study differed largely from other African studies and approximates to an Indian study $(83.7 \%)^{17,18}$. The high rate was found to be associated with the severity of motor function in our study. It may also be attributed to the lower rate of attending school and higher rate of cognitive impairment. In a Sweden study, it was shown that communication function correlates to gross and fine motor and cognitive function and children with severe form of $\mathrm{CP}$ found to have lower communication function level ${ }^{19}$.

The high numbers of severe (quadriplegic) bilateral spastic type of CP suggests injuries to the full term brain due to complications during the birth process such as birth asphyxia or acquired central nervous system infections such as meningitis or encephalitis ${ }^{4}$. The lower frequencies of milder levels of bilateral (diplegic) spastic type of CP suggests injuries to the preterm brain and may reflect less recruitment from children born preterm similar to the findings in Uganda ${ }^{6}$ and contrary to the Australian study where $43 \%$ of children with $\mathrm{CP}$ were born preterm ${ }^{12}$. Probably, children born preterm do not survive the neonatal period due to complications during the first neonatal weeks and limited support from the maternal/newborn health care service provided ${ }^{20}$. This is supported by only few preterm born children in this study, no one with Extremely Low Birth Weight.

On assessment of perinatal factors, we identified that those children with spastic subtype of CP had higher rate of fetal distress and PROM during the perinatal period, higher rate of language and speech difficulty and worse functional impairment while those with Dyskinetic and Ataxic CP were found to have higher rate of precipitated labor and deep jaundice during the neonatal period. These findings may indicate that spastic subtype of cerebral palsy may be associated with perinatal hypoxic insult as in cases of fetal distress, while dyskinetic and ataxic forms may be associated with bleeding and injuries to the deep grey matters of the brain that can occur in cases of precipitated labor ${ }^{20}$. However, due to the fact that the 
possible causes were identified based on history given by the parents (no registries available nor MRI results) causal relationship of definitive risk factors cannot be determined based on this study.

By applying stricter criteria and procedures for setting the diagnosis $\mathrm{CP}$ we found that 22 children of the 176 children who had been given the diagnosis at an earlier visit to the clinic, did not fulfill the SCPE criteria. We also changed the subtype of 20 children following proper evaluation. Over the years the criteria for the $\mathrm{CP}$ diagnose including different subtypes has developed and the newer definition ${ }^{21}$ is now used in most HIC. In combination with functional classifications systems for gross and fine motor, and communication, it provides a detailed description of the child which is very useful in clinical practice for planning interventions and for predicting prognosis. It is also very useful in research when comparing different cohorts of children, e.g., from different countries. Previously older systems have been used in Ethiopia, and probably in many countries in sub-Saharan Africa and other LMIC. A previous retrospective study from our hospital TASH showed $48.2 \%$ of unclassified $\mathrm{CP}^{22}$, which differs significantly from the present study. The prospective assessment of the children in this study provided high-quality clinical information that could be systematized. Hopefully, this study, and studies from other countries in sub-Saharan Africa, can pave the wave for this contemporary method to set diagnose and functional limitations, which would also lead to a change to better clinical practice.

\section{Strengths and limitations.}

A strength in this study was the prospective study design using the contemporary international system for setting CP diagnose and severity level of motor impairments and assessing associated impairments, making it possible to compare with other cohorts. The sample was also large enough to perform some statistical analyses, however, causal relationships cannot be determined from our study as it was a cross 
sectional study done at a point in time. We took a cut off age at 2 years while the SCPE recommends 4. We are confident on the diagnosis of $\mathrm{CP}$ but the subtypes might change with time.

A major limitation was that the sample was not representative for the general population but derived from a clinical cohort, in which families had actively searched for services leading to a bias towards more severe forms. Another limitation was that much of the information on risk factors and associated impairments were based on interviews and surveys of the caregivers which were prone to recall bias.

\section{Conclusion}

Most of the children in our study had severe functional impairments and were dependent on the caregivers for their daily living. This is a hard burden on the care givers in addition to the frustration having no access to rehabilitation services. Proper diagnosis, categorizing into the clinical subtypes, assessing motor function and co morbidities are essential for early intervention and follow-up. The study also find that many children had adverse events during the perinatal period, which could be preventable and call for improved maternal and neonatal care. Overall, the efficient management of these children will require a multidisciplinary care team to obtain the best care of their medical and psychosocial needs and following the ICF frame work $^{25}$.

\footnotetext{
Abbreviations

AAU;Addis Ababa University, CHS;College of Health Science, CP;Cerebral Palsy, GDD;Global Developmental Delay,GMFC;Gross Motor Functional Classification, HIC;High Income Countries, ICF;International Classification of Functioning, Disability and Health, LMIC;Low and Middle Income Countries, PROM;premature Rupture Of Membrane,PNC; Pediatrics Neurology Clinic, SCPE;surveillance of Cerebral Palsy in Europe, TASH;TikurAnbessa Specialized Hospital

\section{Declarations}

Ethical approval and consent

Ethical clearance was obtained from IRB of Pediatrics and Child Health Department's Research and Publications Committee of the School of Medicine, CHS and AAU and all methods were performed in accordance with the relevant guidelines and regulations. Respondents were clearly informed about the purpose of the study and the information required from them. Informed, written consents from primary care takers (parents/guardians) were obtained. Personal identifiers like name of participants were not used to maintain confidentiality. All participants included in the study are kept anonymous during subsequent analysis and dissemination. Children with unaddressed associated impairment diagnosed during the study were linked to the respective specialty evaluation and management.
}

Consent for Publication; Not applicable.

Availability of data and materials; 
The data sets used and/ analyzed during this study are available from the corresponding author on reasonable request.

\section{Competing interests;}

The authors declare that they have no competing interests.

Funding; The study is supported by the neuropediatric unit at Karolinska Institute and the funder Barnhusstiftelsen Frimurare Barnhuset, Department of women's and Children's health.

\section{Acknowledgment}

The researchers would like to gratitude the respondents who participated in the assessment; the two data collectors, Dr Tegegn Molla for statistical analysis of the data and the nurses at the pediatrics neurology clinic for their heartfelt assistance in selection of Patients with pre diagnosis of CP and Ato zeray Berhanu and my parents(Tsige, Kidan and Askalu) for their continuous support.

\section{Authors' contribution;}

ST designed the study, extracted the data, provided oversight of field work and data quality control, interpreted the analysis and finally drafted and wrote the manuscript. HF arranged a visit to Ethiopia to organize the set of data that was collected and he convinced the neuropaediatric unit at Karolinska Institute to fund the research. He was in fact was the basis for ST to pick the research topic seeing what he and his group did at Uganda. Ayalew M organized the pediatrics neurology clinic staff for the data collection and provided confirmatory diagnosis in doubtful cases. HF and Amha M contributed to the design and conceptualization of the study; provided critical input at each report and finding, participated in drafting and improving the manuscript and reviewed the final manuscript as submitted. WA participated on designing the study and reviewing the first report and also helped in the printing of all of the questionnaires used to collect the data. All authors approved the final manuscript as submitted and agree to be accountable for all aspects of the work.

\section{Authors' information;}

Dr Selamenesh Tsige is a consultant pediatrician and an assistant professor in the department of pediatrics and child health at TASH, school of medicine, college of health science, AAU, Addis Ababa, Ethiopia. She is also founder and president of Ethiopian residence community-based NGO called Gojo accommodation and temporary shelter for patients in need, Communication head of East African Academies of Childhood Disability (EAACD) and EAACD representative of GPEC(Global Professional Educational Collaboration),IAACD(International Alliance of Academies of Childhood Disability)

Dr Ayalew Moges is a Consultant Pediatrician, Pediatric Neurologist and an assistant Professor of Pediatrics and Child Health at TASH, school of medicine, college of health science, AAU, Addis Ababa, Ethiopia.

Professor Amha Mekasha (MD, MSC) is a consultant Pediatrician and Professor of Pediatrics and Child Health at TASH, school of medicine, college of health science, AAU, Addis Ababa, Ethiopia.

Dr Workeabeba Abebe(MD,MPH) is a Consultant Pediatrician, Specialist in Pediatric Infectious diseases and an associate Professor of Pediatrics and Child Health at TASH, school of medicine, college of health science, AAU, Addis Ababa, Ethiopia. Professor Hans Forssberg is a Professor in Neuroscience at Karolinska Institute and Consultant in Neuropaediatrics at Astrid Lindgren Children's Hospital. He is also President of International Alliance of Academies of Childhood Disability.

\section{Reference}

1. Diane Damiano, Hans Forssberg. Poor data produce poor models: children with developmental disabilities deserve better. Commentary on Global Burden of Disease (GBD) for developmental disabilities (Lancet Glob Health 2018; 6: e1100-21. October, 2018). www.thelancet.com/lancetgh Vol 7 February 2019.

2. El Tallawy HN, Farghaly WM, Rageh TA, Shehata GA, Metwaly NA, Abo ElftoN,Et al. Epidemiology of major neurological disorders project in Al KhargaDistrict, New Valley. Egypt Neuroepidemiology. 2010; 35:291 - 7. 
3. Couper J. Prevalence of childhood disability in rural kwazulu-Natal. S AfrMed J. 2002; 92:549-52.

4. Kakooza-Mwesige A, Forssberg H, Eliasson AC, Tumwine JK. Cerebral palsy in children in Kampala, Uganda: clinical subtypes, motor function and co-morbidities. BMC Research Notes .2015 ;8: 166. Doi: 10.1186/s13104-015-1125-9.

5. Osama Abas, FatenAbdelaziem, AymanKilany. Clinical Spectrum of Cerebral Palsy and Associated Disability in South Egypt: A Local Survey Study. Open Access Maced J Med Sci. 2017 Mar 15; 5(1):37

6. Angelina Kakooza-Mwesige, Carin Andrews, Stefan Peterson, Fred WabwireMangen, Ann ChristinEliasson, Hans Forssberg. Prevalence of cerebral palsy in Uganda: a population-basedstudy. Lancet Glob Health 2017. http://dx.doi.org/10.1016.

7. Gulam Khandaker, Mohammad Muhit, Tasneem Karim, Hayley Smithers-Sheedy, Iona Novak, Cheryl Jones, Nadia Badawil. Epidemiology of cerebral palsy in Bangladesh: a population-based surveillance study. DMCN 2018. DOI: 10.1111/dmcn.14013

8. SCPE. Surveillance of cerebral palsy in Europe: a collaboration ofCerebral palsy surveys and registers. Dev Med Child Neurol. 2000; 42:816-24

9. Andrews C, Kakooza-Mwesige A, Almeida R, Swartling Peterson S, Wabwire-Mangen F, Eliasson AC, et al. Impairments, functional limitations, and access to services and education for children with cerebral palsy in Uganda: a population-based study. Dev Med Child Neurol. 2019

10. African Child Policy Forum. The Lives of Children with Disabilities in Africa: A Glimpse into A Hidden World. Addis Ababa:(2011).

11. MulugetaBayisaChala, Solomon Mekonnen, GashawAndargie, YigzawKebede, MezgebuYitayal,KassahunAlemu, etal. Prevalence of disability and associated factors in Dabat Health and DemographicSurveillance System site, northwest Ethiopia. BMC Public Health. 2017; 17:762.

12. Group ACPR. Australian Cerebral Palsy Register report. 2016

13. Angelina kakooza-mwesige. Cerebral palsy in Mulago hospital, Uganda:comorbidity, diagnosis and cultural adaptation of an assessment tool. BMC research notes. Stockholm 2016.

14. David R. Bearden, BaphalengMonokwane,EshaKhuranad, James Baierd, Esther Baranove, Kate Westmoreland, LoetoMazhani, and Andrew P. Steenhoff. Pediatric Cerebral Palsy in Botswana: Etiology, Outcomes, and Comorbidities. Pediatr Neurol. 2016 June ; 59 : 23-29.

15. Moifo B, Nguefack, Zeh OF, Obi FA, Tambe J, Mah E, MbondaE,GonsuFotsin J. Computed Tomography Findings In Cerebral Palsy In Yaounde -Cameroon. J AfrImagMéd. 2013; 3(5): 134-142.

16. Singhi P, Saini AG. Changes in the clinical spectrum ofCerebral palsy over two decades in North India - an analysis of 1212 cases.J Trop Pediatr 2013; 59: 434-40.

17. RaghavendraswamiAmoghimath, Vykuntaraju K Gowda, AshaBenakappa. Comorbidities and their relationship to subtype of cerebralpalsy in a tertiary care hospital in South India. Indian Journal of Cerebral Palsy 2017. DOI:10.4103/2395-4264.204408

18. Singhi P, Saini AG. Changes in the clinical spectrum ofCerebral palsy over two decades in North India - an analysis of 1212 cases.J Trop Pediatr 2013; 59: 434-40.

19. Himmelmann K, Lindh K, Hidecker MJ. Communication ability in cerebral palsy: a study from the CP register of western Sweden. Eur J Paediatr Neurol. 2013;17(6):568-74

20. Kenneth F. Swaiman. Pediatric Neurology principles and practice. $5^{\text {th }}$ ed. British Library: Elsevier Saunder; 2012. 2282 p.

21. Peter Rosenbaum. The Definition and Classification of Cerebral Palsy.2008

22. Ayalew Moges, SisayGizaw, GutaZenebe, Suresh Kotagal. Pattern Of Neurological Disorders At Pediatric Outpatient Neurologic Services At TikurAnbessa Specialized Hospital. Ethiop J. Pediatr. Child Health.201; 14(2).

23. Banskota B, Shrestha S,Rajbhandari T, BanskotaAK,Spiegel DA, J Nepal. A Snapshot of 1001 Children Presenting withCerebral Palsy to a Children's Disability Hospital. Health Res Counc. 2015 Jan - Apr;13(29):31-7

24. EDHS 2016

25. WHO International classification of functioning, disability and health : ICF.2001. 



\section{Figures}

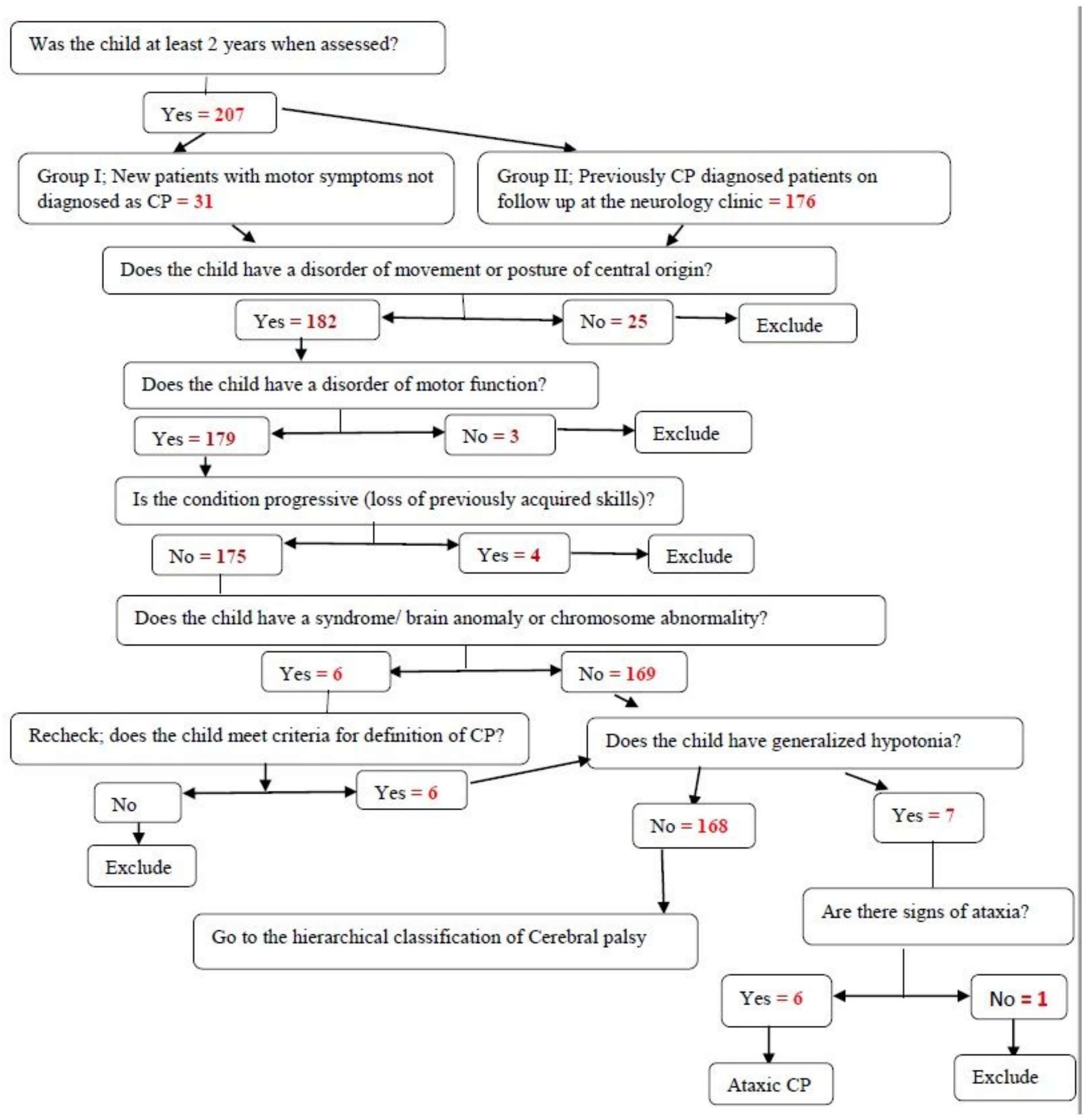

\section{Figure 1}

the process undertaken to select the final participants on the study. Among the initial 207 patients, 174 were included on the final study after excluding the rest based on the SCPE decision tree (Surveillance of cerebral palsy in Europe: a collaboration of Cerebral palsy surveys and registers. Dev Med Child Neurol. 2000; 42:816-24) depicted above. 


\section{Supplementary Files}

This is a list of supplementary files associated with this preprint. Click to download.

- EthiopiaCPmanuscriptsupplementsApril2021.pdf

- EthiopiaCPmanuscriptTablesApril2021.pdf 